\title{
Radiofrequency thermocoagulation of the thoracic splanchnic nerve in functional abdominal pain syndrome -A case report-
}

\author{
Ji-Won Choi ${ }^{1}$, Eun-Young Joo ${ }^{1}$, Sang-Hyun Lee ${ }^{1}$, Chul-Joong Lee ${ }^{2}$, Tae-Hyeong Kim ${ }^{1}$, and Woo-Seok Sim ${ }^{1}$ \\ ${ }^{1}$ Department of Anesthesiology and Pain Meidicine, Samsung Seoul Hospital, Samsung Medical Center, Sungkyunkwan University \\ School of Medicine, ${ }^{2}$ ZEIN (zeropian) Pain Treatment Clinic, Seoul, Korea
}

The thoracic splanchnic nerve block has been used in managing abdominal pain, especially for pains arising from abdominal cancers. A 27-year-old male patient who had a constant abdominal pain was referred to our clinic for pain management but had no organic disease. The numeric rating scale (NRS) for pain scored 7/10. We applied a diagnostic thoracic splanchnic nerve block under the diagnosis of functional abdominal pain syndrome. Since the block reduced the pain, we applied a radiofrequency thermocoagulation at the T11 and T12 vertebral level. Thereafter, his symptoms improved markedly with pain decreasing to an NRS score of $2-3 / 10$. Hereby, we report a successful management of functional abdominal pain via radiofrequency thermocoagulation of the thoracic splanchnic nerves. (Korean J Anesthesiol 2011; 61: 79-82)

Key Words: Functional abdominal pain syndrome, Radiofrequency thermocoagulation, Thoracic splanchnic nerve block.

\begin{abstract}
Mainly for their role in pain management, thoracic splanchnic nerve and celiac ganglion blocks have been of anatomical and clinical interest for many years in the fields of abdominal cancers including the pancreas and stomach in addition to pancreatitis [1]. However they can also be applied to patients suffering from chronic nonmalignant abdominal pain [2]. Radiofrequency ablation is a percutaneous, minimally invasive technique that has been in clinical use for over 25 years. The heat production associated with this technique is neuroablative. Radiofrequency neurolysis has been used to block pain
\end{abstract}

transmission for many kinds of chronic pain that respond to local anesthetics, and it can be expected to prolong the duration of pain relief [3].

We report a case of thoracic splanchnic nerve block and radiofrequency thermocoagulation (RFTC) on a patient with chronic upper abdominal pain.

\section{Case Report}

A 27 -year-old man $(173 \mathrm{~cm}, 68 \mathrm{~kg})$ was referred from the

Received: November 1, 2010. Revised: 1st, December 30, 2010; 2nd, January 19, 2011. Accepted: January 19, 2011.

Corresponding author: Chul-Joong Lee, M.D., Ph.D., ZEIN (zeropain) Pain Treatment Clinic, 35-5, Seongbuk-dong, Seongbuk-gu, Seoul 136822, Korea. Tel: 82-2-763-7533, Fax: 82-2-763-7530, E-mail: ccjlee.mdphd@gmail.com

(c) This is an open-access article distributed under the terms of the Creative Commons Attribution Non-Commercial License (http:// creativecommons.org/licenses/by-nc/3.0/), which permits unrestricted non-commercial use, distribution, and reproduction in any medium, provided the original work is properly cited. 
department of internal medicine to our pain management center for abdominal pain. He described the pain as being continuous and cramping in addition to being localized in the left lower quadrant. On physical examination, he had no tenderness in the abdomen. Large meals worsened his pain but the pain did not fluctuate in intensity. He had no change of bowel habits or sleep disorders. He stated that his symptoms had started with epigastric discomfort and soreness 7 to 8 years ago and was variable thereafter. He had received medical treatments at local internal and oriental medical clinics without a significant improvement and then visited this hospital 2 years ago. His numeric rating scale (NRS) for pain scored 7/10 (7 out of NRS scale of 10 [0: no pain, 10: imaginable worst pain]).

He had no other chronic disease, did not smoke or drink alcohol. The results of an esophagogastroduodenoscopy taken at this hospital 2 years ago had no specific findings except for chronic superficial gastritis. Abdominal ultrasonographic and blood tests were normal. Colonoscopic findings at a local clinic 16 months ago were also normal. He was prescribed proton pump inhibitors, antacids, digestants and prokinetics intermittently, but these had no beneficial effects. Five months ago, he had abdominal and pelvic computerized tomography, but there were no significant findings except for two simple cysts in the right kidney.

We decided to perform a diagnostic left thoracic splanchnic nerve block under the diagnosis of functional abdominal pain syndrome (FAPS). Informed consent for the block was obtained from the patient after explaining the purpose of and what was expected from the procedure and its potential complications. With the patient in the prone position, a pillow was inserted under the abdomen to reduce lumbar kyphosis. Electrocardiogram, pulse oximetry, and arterial blood pressure were monitored during the procedure, and periprocedural intravenous hydration was performed using $0.9 \%$ saline solution. No intraoperative sedation was administered. $10 \%$ povidone-iodine (Besetine ${ }^{\circledR}$, Hyundaipharm, Korea) and $75 \%$ alcohol were used to sterilize the area of interest. The fluoroscope was used to identify the T11 vertebral body and was rotated obliquely approximately 15 degrees toward the ipsilateral side, squaring up the T11 vertebral body. After the needle entry site and expected subcutaneous needle trajectory were anesthetized using 1\% lidocaine, a Quincke type, 23 gauge, 12-cm spinal needle (Hakko Co., Naganoken, Japan) with manually bended tip was advanced by means of a tunnel vision technique toward the lateral margin of the vertebra in oblique projection. After the needle touched the lateral margin of the vertebral column, the needle was advanced to the junction between the anterior third and middle third of the T11 vertebral body with the lateral projection, hugging the lateral edge of the T11 vertebral body. Two $\mathrm{ml}$ of contrast agent (Iobrix ${ }^{\circledR}, 300 \mathrm{mg} \mathrm{I} /$ $\mathrm{ml}$, Accuzen, Korea) was injected to confirm compartment flow of the injectate and to rule out any intravascular or intrapleural spread under real time fluoroscopy. After confirmation, $4 \%$ lidocaine $(4 \mathrm{ml})$ was injected.

Ten minutes later, the patient reported relief of symptoms with a decrease in NRS score from 7 to 3 . After assuring that he had no neurologic deficits, such as sensory or motor dysfunctions, and no hypotension, he got a chest radiograph to rule out a possible pneumothorax related to the procedure. He had no abnormal findings in the chest radiograph. Two weeks later, he revisited and told that abdominal pain has relapsed in a few hours after the nerve block. We evaluated that the block was successful and ruled out a psychogenic effect. We recommended for a second diagnostic nerve block, however he refused to undertake another diagnostic block and requested a procedure with a prolonged effect. We decided to perform radiofrequency thermocoagulation of the left thoracic splanchnic nerves at the T11 and T12 vertebral level. The patient position and skin preparation were the same as in the previous thoracic splanchnic nerve block. Two disposable 20
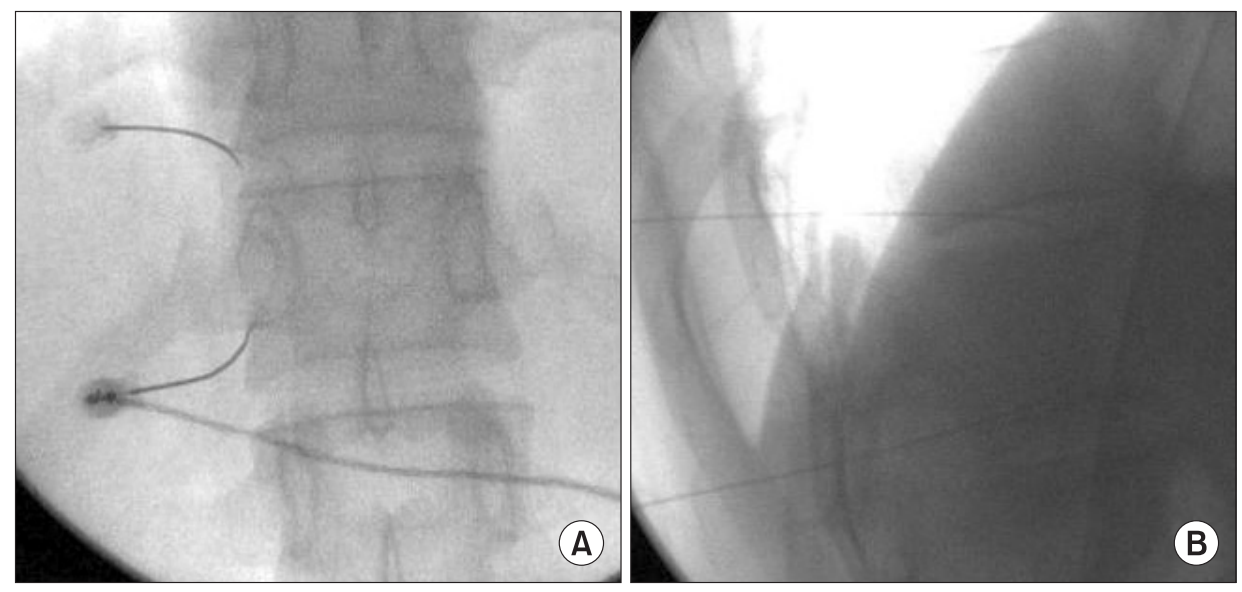

Fig. 1. The tips of radiofrequency cannulas are located at the junction of the anterior and middle thirds of the T11 and T12 vertebral body. Anteroposterior (A) and lateral (B) fluoroscopic images. 
gauge $145 \mathrm{~mm}$, curved-tipped radiofrequency cannulas (BMC RF CANNULA ${ }^{\circledR}$, BMC Inc, QC, Canada) with a $10 \mathrm{~mm}$ active tip were advanced to the junction between the anterior third and middle third of the T11 and T12 vertebral body with the lateral projection using the same method as described for the nerve block. Once the cannulas were in place, we performed nerve stimulations. At $50 \mathrm{~Hz}$, the sensory stimulation was conducted up to $1 \mathrm{~V}$ at each level and the patient reported some discomfort at the epigastrium and the original abdominal pain area. There was no sign of stimulation of the T11 or 12 nerve root. At $2 \mathrm{~Hz}$, the motor stimulation was increased to 2 $\mathrm{V}$ and we confirmed no contraction of the intercostal muscles (Fig. 1). After $2 \mathrm{ml}$ injections of $2 \%$ lidocaine at each level, we produced RFTC lesioning at $80^{\circ} \mathrm{C}$ for 90 seconds. Then, we rotated the curved-tipped cannulas and performed the thermocoagulation lesioning again to obtain the larger lesion. After lesioning, triamcinolone $(20 \mathrm{mg}$ ) was injected at each level. After completion of the procedure, he reported pain relief as $2-3 / 10$ on the NRS. We observed the patient closely for 30 minutes and had him take a chest radiograph to check a possible pneumothorax. He was discharged with his mother after confirmation of no complications.

During an eight week follow up, he reported that his reduction in pain was sustained.

\section{Discussion}

FAPS is a relatively uncommon functional gastrointestinal disorder defined by the presence of constant or frequently recurring abdominal pain that is not associated with eating, change of bowel habits or menstrual periods and points to a more centrally targeted basis for the symptoms [4]. Further, it has a higher co-morbidity with psychiatric disorders [4]. The etiology and pathophysiology are incompletely understood and the diagnostic criteria has been based on the Rome III Multiconsensus Group in 2006 [4]. In the U.S householder study of functional gastrointestinal disorders, FAPS was seen in $2 \%$ of respondents, compared with $9.2 \%$ for irritable bowel syndrome [5]. FAPS has been mainly discussed in the pediatric field where it is most experienced by children between the ages of $8-15$ years with the most common age of onset being 9.5 years [6]. In our case, the patient began to get the symptoms in his second decades.

In subjects with FAPS, an almost universal finding is that they experience more pain than normal subjects in response to an experimental visceral stimulation. This is termed visceral hypersensitivity [7]. The 'brain-gut connection' suggests that the patient with FAPS has differences in their central and enteric nervous systems, which cause bowel hyper-reactivity by stimuli and results in abdominal pain [7]. Most researchers and clinicians agree that it is of multi-factorial etiology coupled with an altered brain-gut interaction [7].

Thoracic splanchnic nerve block has been used to manage upper abdominal pain related to malignancies or chronic benign abdominal pain syndromes such as chronic pancreatitis [1]. There had been attempts, several decades ago, to use this block to treat gastric and duodenal ulcers [8]. In patients with chronic abdominal pain, interruption of the celiac plexus or thoracic splanchnic nerves can offer symptomatic pain relief by inhibiting nociceptive pathways from the abdominal viscera to the brain. Recently the thoracic splanchnic nerve block has gained a renewed interest because the thoracic splanchnic nerve sits in a less variable anatomical spatial relationship with other surrounding structures, which yields a highly predictable needle trajectory while performing the nerve block compared to the conventional celiac plexus block [9]. In the present case, the patient complained of lower abdominal pain. The superior hypogastric plexus is a more commonly blocked structure for lower abdominal pain, but since the thoracic splanchnic nerves innervate a wide range of abdominal organs, the thoracic splanchnic nerve block could also be considered relevant to the patient. Moreover, this block is less invasive than the superior hypogastric plexus block. Therefore, we selected a thoracic splanchnic nerve block as our primary choice. In case of treatment failure, we had planned to perform a superior hypogastric plexus block.

At first visit, the patient underwent a test block of the thoracic splanchnic nerves to ensure improvement in his pain level. The block was effective, and we performed RFTC within two weeks. Although there is no consensus as to the number of diagnostic blocks before performing a permanent neurolysis, we considered at least two trial nerve blocks before neurolysis would be a better strategy to rule out a false positive treatment effect. However, we performed the RF ablation on this patient after one trial, a diagnostic thoracic splanchnic block. In the diagnostic block, pain relief lasted only for the local anesthetic's duration of action; therefore, we agreed that the pain relief originated from an effective thoracic splanchnic block. Moreover, our patient strongly requested for an immediate therapeutic block with a prolonged duration of effect.

Traditionally, thoracic splanchnic nerve neurolysis is performed with $\sim 10 \mathrm{ml}$ of absolute alcohol or $6-10 \%$ phenol. However, neurolysis with the above chemical agents are marred by the difficulty in predicting its spread within the anatomical compartment containing the thoracic splanchnic nerves. The diffusion of agent may cause secondary pain caused by nerve root damage, or could damage the surrounding vascular structures such as the aorta or vena cava [10]. But, RFTC is exempt of such complications as well as being minimally invasive and a relatively safer technique. Another advantage 
of RFTC is that it has an immediate effect unlike alcohol and phenol, which may take up to 1 week or 10 days to achieve neurolysis [9]. Due to these advantages of RFTC, there has been growing interest in the use of this technique for neurolysis of the splanchnic nerves [9]. However, there is lack of data on RFTC of the thoracic splanchnic nerves or its use in FAPS.

Marra et al. [11] reported that thoracic splanchnic nerve blocks are associated with a better success rate than the celiac plexus block. Raj et al. [12] reported that up to $40 \%$ of patients had excellent pain relief after a thoracic splanchnic nerve block, with only a $15 \%$ of patients achieving poor results in a series of 107 patients with abdominal pain. In a paper by Garcea et al. [9], which included eight patients with chronic pancreatitis and two with chronic abdominal pain of uncertain etiology, thoracic splanchnic nerve RFTC resulted not only in a decrease of pain scores, opiate analgesia use and acute admissions for pain control, but it also resulted in improvement of other parameters associated with long term debilitating chronic pain such as anxiety levels, daily activity, overall mood and general perception of health.

Treatment recommendations for patients with FAPS are empirical and not based on results from well-designed clinical trials. The accepted basis for clinical management of FAPS relies on establishing an effective patient-physician relationship, following a general treatment approach and offering more specific management that often encompasses a combination of treatment options [13]. Therefore, generally, the treatment of FAPS includes pharmacological, psychological and complementary therapies. Psychological disorders such as anxiety and depression are common in patients with FAPS [14]. We could not obtain our patients psychiatric medical history. The internal medicine doctor had advised him to visit a psychiatrist, however he refused. Because he was discouraged with the failures of conservative therapies, he might have benefited from a psychiatric treatment such as cognitivebehavioral therapy along with our FAPS management [14]. Multidisciplinary pain treatment provides comprehensive rehabilitation of patient with chronic pain. And it might be the most effective method of treating disability from refractory chronic pain symptoms [14].

Another concern in this case is the possibility of underdiagnosing structural diseases, especially cancer. Several retrospective and prospective studies suggest that diagnostic failures occur in only $0 \%$ to $4.5 \%$, but this should be kept in mind in the follow up of such patients, especially if symptoms change [15]. Therefore, in managing these patients, the pain specialists should always be aware of possible concurrence or progression of another disease depending on change in symptoms.

In conclusion, pain control is one of the most important components in the management of FAPS and the thoracic splanchnic nerve block and radiofrequency thermocoagulation can be used for this purpose.

\section{References}

1. Loukas M, Klaassen Z, Merbs W, Tubbs RS, Gielecki J, Zurada A. A review of the thoracic splanchnic nerves and celiac ganglia. Clin Anat 2010; 23: 512-22.

2. Malfertheiner P, Domínguez-Muñoz JE, Büchler MW. Chronic pancreatitis: management of pain. Digestion 1994; 55: 29-34.

3. Racz GB, Ruiz-Lopez R. Radiofrequency procedures. Pain Pract 2006; 6: 46-50.

4. Drossman DA. The functional gastrointestinal disorders and the Rome III process. Gastroenterology 2006; 130: 1377-90.

5. Drossman DA, Li Z, Andruzzi E, Temple RD, Talley NJ, Thompson WG, et al. U.S. householder survey of functional gastrointestinal disorders. Prevalence, sociodemography and health impact. Dig Dis Sci 1993; 38: 1569-80.

6. Scholl J, Allen PJ. A primary care approach to functional abdominal pain. Pediatr Nurs 2007; 33: 247-54.

7. Aziz Q, Schnitzler A, Enck P. Functional neuroimaging of visceral sensation. J Clin Neurophysiol 2000; 17: 604-12.

8. Stojic B. Treatment of gastric and duodenal ulcer by splanchnic nerve block. Med J Aust 1969; 22: 654-6.

9. Garcea G, Thomasset S, Berry DP, Tordoff S. Percutaneous splanchnic nerve radiofrequency ablation for chronic abdominal pain. ANZ J Surg 2005; 75: 640-4.

10. Wong GY, Schroeder DR, Carns PE, Wilson JL, Martin DP, Kinney $\mathrm{MO}$, et al. Effect of neurolytic celiac plexus block on pain relief, quality of life, and survival in patients with unresectable pancreatic cancer: a randomized controlled trial. JAMA 2004; 291: 1092-9.

11. Marra V, Debernardi F, Frigerio A, Menna S, Musso L, Di Virgilio MR. Neurolytic block of the celiac plexus and splanchnic nerves with computed tomography. The experience in 150 cases and an optimization of the technique. Radiol Med 1999; 98: 183-8.

12. Raj PP, Sahinler B, Lowe M. Radiofrequency lesioning of splanchnic nerves. Pain Pract 2002; 2: 241-7.

13. Chang L, Drossman DA. The psychosocial interview in the irritable bowel syndrome. Clin Perspect Gastroenterol 2002; 5: 336-41.

14. Drossman DA. Functional abdominal pain syndrome. Clin Gastroenterol Hepatol 2004; 2: 353-65.

15. Harvey RF, Mauad EC, Brown AM. Prognosis in the irritable bowel syndrome: a 5 year prospective study. Lancet 1987; 329: 963-5. 